\title{
A GEO-LEO Hybrid Architecture Design of Satellite Internet
}

\author{
Yingyuan Gao ${ }^{\mathrm{a},}$, , Siqian Cui ${ }^{\mathrm{b}}$, Zhou Lu \\ China Academy of Electronics and Information Technology, Beijing, 100041, China \\ agyy_0303@163.com, ${ }^{b}$ csq_0303@163.com, ccete_luzhou@163.com \\ *Gao Yingyuan
}

Keywords: Satellite internet constellation, Internet broadband access, GEO-LEO hybrid network.

Abstract: This paper analyzed the development course, present situation and trend of traditional satellite communication system which can provide internet broadband access and emerging satellite internet constellation. Considering the unique advantages of the geostationary earth orbit (GEO) and low earth orbit (LEO) satellite system and our country's national conditions, the concept of GEO-LEO hybrid satellite internet constellation is put forward, which provides the construction suggestions for our country's development of satellite internet constellation.

\section{Introduction}

In recent years, satellite internet has become an inevitable development trend which combined satellite communication and internet. Looking back at the history of satellite internet development, we can find that the satellite constellation that provides internet services is not a new thing. It has a history of nearly 30 years of development. However, it is slow to develop, some have not been implemented, some have died, and some applications are limited.

Under the promotion and support of internet giants such as Google and Facebook, innovative companies such as Space X and OneWeb have planned to build a constellation system consisting of numerical LEO small satellites to actively seize new resources in space internet access, which also trigger a global development boom.

This paper focuses on the concept connotation, development overview, trends and challenges of satellite internet, and proposes our country's construction plan which is based on our county's practical situation. Furthermore, the paper also analyzes the key issues should be considered of the construction plan.

\section{The Development Status of Foreign Satellite Internet}

At present, both traditional satellite communication companies and emerging internet commercial 
companies are paying great attention to the development of broadband satellite communication. In the following, this paper describes the development status of foreign satellite internet from three aspects: GEO,medium earth orbit(MEO) and LEO.

\subsection{GEO Broadband Satellite Communication System}

\subsubsection{IPSTAR ${ }^{[1]}$}

IPSTAR is the world's largest communications satellite launched in August 2005. It provides broadband services such as multimedia broadcasting, broadband network access, and video conferencing for users in 22 countries and regions in the Asia-Pacific region. The satellite uses the $\mathrm{Ku} / \mathrm{Ka}$ hybrid band to provide Ku-band spot beams (84), Ku-band shaped beams (3), regional broadcast beams (7) and 18 Ka-band spot beam coverage for the Asia-Pacific region. The total bandwidth of the system is $45 \mathrm{Gbps}$, of which $12 \mathrm{Gbps}$ covers the whole of China.

\subsubsection{Broadband Global Area Network(BGAN) ${ }^{[2]}$}

The Broadband Global Area Network is a global satellite broadband LAN based on Inmarsat-4 satellite. The system operating frequency band is in the L-band, the downlink rate is $216 \mathrm{kbps} \sim 432 \mathrm{kbps}$, and the uplink rate is $72 \mathrm{kbps} \sim 432 \mathrm{kbps}$, which realizes the conversion from analog to digital, from traditional circuit switching to Internet service, and from narrowband voice data to broadband high-speed data. The satellite system can cover $85 \%$ of the world's land area, providing mobile users with video broadcast, broadband network access and other services.

\subsubsection{Spaceway-3 ${ }^{[3]}$}

Developed and operated by Hughes Network Systems, the Spaceway-3 satellite communications system was launched in 2007 and is the world's first satellite with on-orbit switching and routing capabilities. Spaceway-3 significantly reduces network transmission delays by using Ka-band, multi-beam and on-board fast packet switching technology, covering all of the United States and most of Canada. The total communication capacity of the system is $10 \mathrm{Gbps}$, which can accommodate 1.65 million user terminals, and the capacity is 5-8 times that of Ku-band communication satellites.

\subsubsection{ExeDe Internet ${ }^{[4]}$}

ExeDe Internet consists of ViaSat-1 and ViaSat-2 broadband communication satellites, which were launched in 2011 and 2017 respectively. It is currently the largest GEO broadband satellite communication system. ViaSat-1 uses Ka-band spot beam technology with a total capacity of 140 Gbps and a download rate of $12 \mathrm{Mbps}$, which can meet the satellite Internet access needs of more than 2 million users. The ViaSat-2 satellite is the largest satellite ever launched by Boeing. It has a full-star capacity of $300 \mathrm{Gbps}$ and covers seven times the coverage of ViaSat-1, providing up to 25 Mbps of broadband service to 2.5 million subscribers. In addition, Viasat-3 is scheduled to be launched in 2019. It consists of three satellites, each with a capacity of 1 Tbps.

\subsection{MEO Satellite Internet Constellations}

The MEO satellite internet constellation is mainly represented by the O3b plan [5].

O3b, short for the other 3 billion, the plan is to solve the internet problem of the remaining 3 billion people who have failed to access the internet due to geographical and economic factors. It is proposed 
by O3b Networks Corporation. O3b has successfully deployed 16 MEO satellites since June 2013, covering a total of seven regions, using the Ka-band, with a single-star throughput of approximately 16 Gbps. Currently, 16 satellites were fully operational, providing service capabilities with a relay bandwidth of $600 \mathrm{Mbps}$ and a latency of no more than $150 \mathrm{~ms}$. Since 2017, O3b have begun next generation satellite constellation plan-O3b mPower, which will realize global coverage.

\subsection{LEO Satellite System}

\subsubsection{Traditional LEO Satellite Communication System-_-Iridium ${ }^{[6]}$}

The first generation Iridium system was completed and commercialized in 1998. It was declared bankrupt in 1999 and was later acquired by the "New Comet" company. The Iridium constellation has an orbital height of $780 \mathrm{~km}$ and consists of 66 satellites. The second generation of Iridium achieves higher service rate, larger transmission capacity and more functions through the gradual upgrade of the first generation syestem. After the deployment of the second generation of Iridium, the transmission rate has been increased to $1.5 \mathrm{Mbps}$, and the speed of transport and portable terminals has been increased to $30 \mathrm{Mbps}, 10 \mathrm{Mbps}$. The second-generation system also has functions such as ground imaging, aviation monitoring, navigation enhancement, and weather monitoring.

\subsubsection{Satellite Internet Constellation}

\section{(1) OneWeb}

The OneWeb constellation was proposed by OneWeb, founded by Greg Wyler who is also the founder of O3b Corporation. It plans to deploy nearly 3,000 LEO satellites, initially using the $\mathrm{Ku}$-band and expanding to the Ka and $\mathrm{V}$ bands. At the beginning of the constellation, it plans to launch 720 satellites with an orbital altitude of $1200 \mathrm{~km}$. It adopts a simple design —— transparent forwarding method to provide internet access services. The weight of the single satellite is no more than 150 kilograms and the capacity is more than 5 Gbps. At the same time, OneWeb has been authorized by the US Federal Communications Commission to provide internet services in the United States. OneWeb plans to launch in 2018 and begins to provide services in 2019.

(2) Starlink

Starlink satellite internet constellation was proposed by Space X. Space X plans to build a constellation of nearly 12,000 satellites, consisting of 4425 low-orbit constellations distributed at a height of $1150 \mathrm{~km}$ and 7518 very low-orbit constellations distributed around $340 \mathrm{~km}$. The low-orbit constellation selects the $\mathrm{Ku} / \mathrm{Ka}$ band to facilitate better coverage; the very low-orbit constellation uses the V-band to achieve signal enhancement and more targeted services. Space $\mathrm{X}$ plans to have such a network cover any location on the earth. Space X expects the system to have more than 40 million users by 2025 and revenues of $\$ 30$ billion. Space $X$ is more focused on satellite manufacturing while operating in the constellation. Therefore, Space $\mathrm{X}$ needs more financing and is expected to need financing of $\$ 10-15$ billion.

\subsection{Analysis of Foreign Satellite Internet Constellations}

Comparing the GEO and LEO systems in terms of system scale, system capacity, and coverage, we can find that they have their own advantages. The LEO satellite internet constellation has obvious advantages in terms of coverage, bridging the digital divide, network delay, and system capacity. The user terminal equipment is easier to achieve miniaturization and hand-held. The GEO satellite communication system has relatively easy frequency coordination, longer operating life, and 
relatively lower system construction and maintenance costs. In addition, although the capacity of the LEO satellite internet constellation system is higher than that of the GEO broadband communication satellite, the GEO satellite has an advantage in transmitting high-bandwidth capacity through point beam (for example, providing high-definition live broadcast services for regional users). Therefore, the construction of the satellite Internet constellation needs to coordinate the superiority of the GEO and LEO satellite systems and achieve complementary advantages.

\section{Conception of Our country's Satellite Internet Construction Plan}

\subsection{The Necessity of Developing Satellite Internet in China}

In the new era, China's demand for global real-time seamless information protection has grown significantly. Facing the current development boom of the world's low-orbit satellite internet constellation, China also needs to seize the opportunity to seize resources and build a satellite internet constellation with independent control and global coverage. However, China's construction of low-orbit satellite internet constellation has the following two limitations. One aspect is the restriction on the global establishment of the ground station. Due to China's national conditions, China cannot use the global ground station to achieve global services for satellite communications. Thus China cannot realize global coverage through only LEO constellation. Another aspect is the frequency orbit resource limit. At present, the international frequency orbital resources are scarce, and the low-orbit frequency resources are being divided up. China's frequency resources are insufficiently accumulated and it is in an inferior position in the world. Therefore, it is difficult to guarantee the construction of the low-orbit constellation.

Therefore, starting from the actual situation in China, the GEO-LEO hybrid satellite internet constellation plan is proposed. This plan can break through the global ground station limit and the lack of frequency resources, making full use the superiority of GEO satellite system, breaking the political geopolitical restrictions, ensuring the security and reliability of the satellite internet business, while providing users with a better service experience.

\subsection{Our Country's Satellite Internet Development Plan}

Based on the above reasons, this paper proposes a GEO-LEO hybrid satellite internet constellation scheme as shown in Figure 1. In this scheme, the low-orbit constellation only has the same-orbit link, and different orbital planes are transited through the high-orbit satellite. Compared with the pure low-orbit scheme, such a networking architecture can improve network connectivity and quality of service while reducing the total number of links in the network.

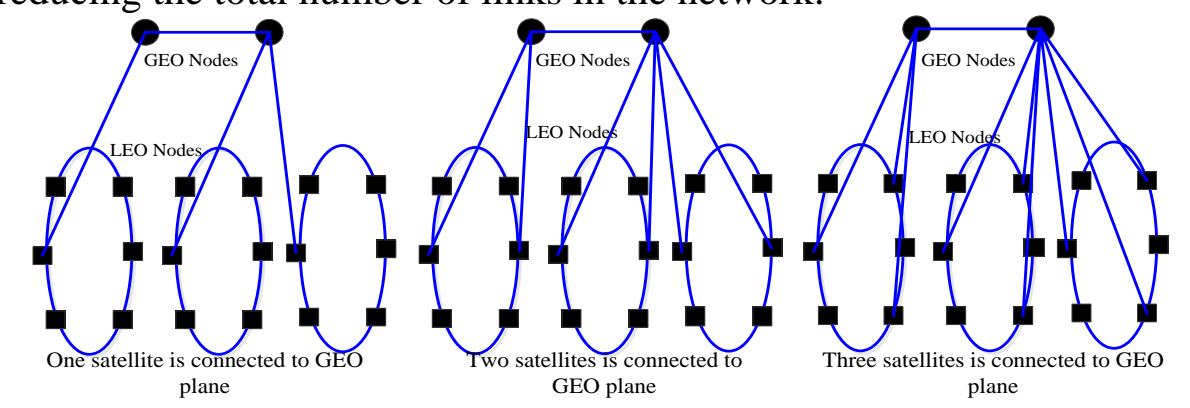

Fig 1. The architecture of GEO and LEO combined satellite internet system

Specifically, the physical architecture, network architecture, and networking service scheme of the proposed scheme are designed as follows. 
Physical architecture: The physical architecture adopts the GEO+composite LEO constellation scheme, in which the main functions of GEO satellites are backbone transmission, large node access services, service allocation, operation management, and relay nodes between low-orbit satellites. The LEO constellation adopts a composite orbit constellation composed of different types of orbital height, dip angle, and ascending node, as shown in Figure 2. Compared with the single orbit constellation, the global orbital scheme formed by the composite orbit constellation is more flexible and the coverage characteristics are more diverse. At the same time, the composite orbit constellation can dynamically allocate services between different types of orbiting satellites based on service demand and traffic within the coverage area.

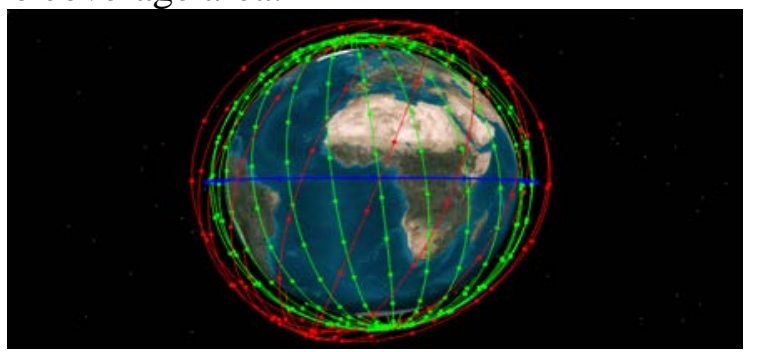

Fig 2. The compounded LEO constellations

Network architecture: It consists of three service networks and one service plane. The service networks are oriented user centers, data centers and information exchange, and the service plane is oriented cloud networks. This new network architecture improved the defections of the old coordination and configuration of space-based network resources in the past, in which the response time of network planning is too long. As this new network becomes a configurable service resource, users can obtain network resources on demand and manage their own virtual network resources to get the services they need.

Network service: It has the ability to acquire, auto-deploy and intelligently tune the resources of the whole network. It fully supports service dynamic programming and network slicing technology based SDN/NFV/MEC, and could collaborative schedule central network, access network, data center and the user network together, to achieve an agile and on-demand response to network topology, channel bandwidth, transmission quality, and latency requirements.

\subsection{Key Issues to be Solved in China's Satellite Internet Constellation Scheme}

In order to realize the proposed GEO-LEO hybrid solution, several key issues must be addressed as follows.

\subsubsection{GEO-LEO network collaborative networking problem}

For the GEO-LEO hybrid constellation system, combined with demand of the ground user access and the integration of the space and ground information network, it is necessary to carry out research on the coordination of GEO and LEO physical layers, to adapt to the large-scale, high-latency, high-dynamic spatial routing protocol. It is also necessary to solve the problem of flexible networking to compensate for the inherent vulnerability of space-based networks and improve the connectivity of entire network in full-time and global.

\subsubsection{Mobility management issues}

Aiming at mian problems of high dynamic motion of LEO constellation nodes and the frequent switching of multi-satellite and multi-beams for users, it is necessary to focus on the key technologies 
research on mobility management based on identity and location separation, multi-beam non-inductive fast switching, adaptive link rate changing, network resource fast scheduling and controlling and so on, which could effectively reduce service delays and improve network mobility management efficiency and service quality.

\subsubsection{Integration with ground networks}

For the integration demand of satellite internet and ground internet, it is necessary to focus on the key technologies research on the heterogeneous protocol's interconnection design of satellite internet and future internet, network slicing based on multi-dimensional network resource virtualization, network control based application-driven, network resource scheduling on-demand and so on, to achieve efficient and reliable interconnection between satellite internet and ground internet.

\subsubsection{Network security issues}

In view of the wide openness, it is necessary to focus on the key technologies research on satellite internet network security assurance system with expansion and evolution capabilities. Meanwhile it is also necessary to study the network security model and the interactive control mechanism to adapt to time and space changes of cyber threaten and diversified tasks, the dynamic response of the security policy, the active adaptation, the seamless migration and the anti-destruction and fault tolerance mechanism, to provide systematic security for the safe and reliable operation of satellite internet.

In addition, in terms of satellite launch manufacturing and operation management, it is necessary to learn from foreign satellite mass production and advanced investment and financing concepts to reduce the satellite development cycle and operating costs.

\section{Conclusion}

With the rapid growth of demand for multimedia services and internet services, countries are beginning to pay more attention to the development of the satellite internet industry. Under the current global satellite internet construction boom, based on our country's aerospace industry and the Internet industry basis, our country should actively seize this huge market and firmly grasp the historical development opportunities.

\section{References}

[1] THESLING W, VANDERAAR M, THOMPSON M, et al. Two-way Internet over iPSTAR using advanced error correction and dynamic links[C].AIAA International Communication Satellite System Conference. Montreal, Canada, 2002.

[2] FEBVRE P, BOUTHORS X, MAALOUF S. Efficient IP-multicast via Inmarsat BGAN, a 3GPP satellite network[J]. Satellite Communication Network, 2007, 25: 459-480.

[3] Feng Shaodong, Feng Qi, Shen Jun. Overview of the US Spaceway3 System [J]. Digital Communication World, 2009 (12): 82-85.

[4] https://www.viasat.com/broadband -satellite -networks/high-capacitysatellite-system[EB/OL].

[5] http://www.o3bnetworks.com/o3b-advantage/our-technology[EB/OL].

[6] STERLING D E, HARLELID E. The Iridium system—a revolutionary satellite communications system developed with innovative applications of technology[C]. Proceedings of IEEE Military Satellite Communications Conference, McLean, America,1991,436-440. 